\title{
A Note on the Minimum Wiener Polarity Index of Trees with a Given Number of Vertices and Segments or Branching Vertices
}

\author{
Sadia Noureen, ${ }^{1}$ Akhlaq Ahmad Bhatti, ${ }^{1}$ and Akbar Ali $\mathbb{D}^{2,3}$ \\ ${ }^{1}$ Department of Sciences and Humanities, National University of Computer and Emerging Sciences, \\ Lahore Campus, B-Block, Faisal Town, Lahore, Pakistan \\ ${ }^{2}$ Department of Mathematics, Faculty of Science, University of Ha'il, Ha'il, Saudi Arabia \\ ${ }^{3}$ Knowledge Unit of Science, University of Management and Technology, Sialkot, Pakistan \\ Correspondence should be addressed to Akbar Ali; akbarali.maths@gmail.com
}

Received 4 July 2020; Revised 2 January 2021; Accepted 11 January 2021; Published 23 January 2021

Academic Editor: Juan L. G. Guirao

Copyright (C) 2021 Sadia Noureen et al. This is an open access article distributed under the Creative Commons Attribution License, which permits unrestricted use, distribution, and reproduction in any medium, provided the original work is properly cited.

\begin{abstract}
The Wiener polarity index of a graph $G$, usually denoted by $W_{p}(G)$, is defined as the number of unordered pairs of those vertices of $G$ that are at distance 3. A vertex of a tree with degree at least 3 is called a branching vertex. A segment of a tree $T$ is a nontrivial path $S$ whose end-vertices have degrees different from 2 in $T$ and every other vertex (if exists) of $S$ has degree 2 in $T$. In this note, the best possible sharp lower bounds on the Wiener polarity index $W_{p}$ are derived for the trees of fixed order and with a given number of branching vertices or segments, and all the trees attaining this lower bound are characterized.
\end{abstract}

\section{Introduction}

A topological index is a numerical quantity calculated from a graph, which remains unchanged under graph isomorphism [1]. Topological indices have attracted much attention in recent years, as many of them provide a good correlation between the molecular structure of a chemical compound and its properties. Examples for calculating the topological indices of particular graphs can be found in [2-4].

The Wiener polarity index $W_{p}$ is one of the oldest topological indices, which was proposed in 1947 by the chemist Harold Wiener [5], for predicting the boiling points of paraffins. The index $W_{p}$ for a graph $G$ is defined as the number of unordered pairs of those vertices of $G$ that are at distance 3 . In the previous decade, $W_{p}$ has attracted much attention from researchers; for example, see the surveys $[6,7]$, papers [8-25], and related references therein.

Before moving further, let us recall some definitions and notations first. All the graphs considered in this note are simple and finite. Let $G$ be a graph with the vertex set $V(G)$ and the set of edges $E(G)$. The degree of a vertex $u \in V(G)$ is denoted by $d_{u}(G)$ (or simply by $d_{u}$ if the graph under consideration is clear). The number of vertices in a graph is known as its order. A graph of order $n$ is called an $n$-vertex graph. A vertex of degree 1 is called pendent vertex, while a vertex of degree greater than 2 is known as a branching vertex. Let $N_{G}(u)$ (or $N(u)$ ) be the set of all those vertices of $G$ that are adjacent to the vertex $u \in V(G)$. As usual, we denote by $P_{n}$ and $S_{n}$ the path and the star graph of order $n$, respectively. A segment $S$ of a tree $T$ is a nontrivial path (that is, a path of length at least 1 ) in $T$ with the property that both the end-vertices of $S$ have degrees different from 2 in $T$ and every other vertex (if exists) of $S$ has degree 2 . A tree $S T$ is called starlike tree (or generalized star) if it contains exactly one branching vertex (we call it the central vertex of $S T$ ). A path $P=v_{0}, v_{1}, \ldots, v_{k}$ in a tree $T$ is called a pendent path (internal path, respectively) of length $k$, if one of the two vertices $v_{0}, v_{k}$ is pendent and the other is branching (both the vertices $v_{0}$ and $v_{k}$ are branching, respectively) and $d_{v_{i}}=2$ if $1 \leq i \leq k-1$. The notation and terminology of (chemical) graph theory that are not defined in this note can be found in [1, 26-28].

By using the definition of the Wiener polarity index, Lukovits and Linert [29] demonstrated the quantitative structure-property relationships in a series of acyclic and cycle-containing hydrocarbons. Considerable work has been 
done, however, on characterizing the trees that maximize or minimize $W_{p}$ under various additional conditions: for example, with given order [15], degree sequence [30, 31], diameter [32], and pendent vertices [33, 34]. Shafique and Ali [35] gave some structural properties of the trees of fixed order and with a given number of segments or branching vertices having maximum/minimum $W_{p}$ value. Here, in this note, we are specifically interested in extending the results obtained in the paper [35].

Du et al. [15] showed that $W_{p}$ of a tree $T$ can be written as

$$
W_{p}(T)=\sum_{u v \in E(T)}\left(d_{u}-1\right)\left(d_{v}-1\right),
$$

where $u v$ is the edge connecting the vertices $u, v \in V(T)$. Here, it is important to note that $W_{p}$ coincides with reduced second Zagreb index [35-37], for the case of trees.

For fixed integers $n$ and $s$, denote by $\mathbb{S} \mathbb{T}_{n, s}$ and $\mathbb{T}_{n, b}$ the classes of all $n$-vertex trees with $s$ segments and $b$ branching vertices, respectively, where $1 \leq s \leq n-1$ and $1 \leq b \leq(n / 2)-1$. In this note, we characterize all the trees attaining minimum $W_{p}$ value from each of the two classes $\mathbb{S} \mathbb{T}_{n, s}$ and $\mathbb{T}_{n, b}$ and hence provide the solution of a problem, left open in [35], concerning the minimum $W_{p}$ value.

Let $T^{\prime}$ be a tree obtained from a tree $T$ after applying a transformation such that $V(T)=V\left(T^{\prime}\right)$. Throughout this note, whenever we consider such trees, by $d_{v}$ and $N(v)$ we mean the degree and set of neighbors, respectively, of the vertex $v \in V(T)=V\left(T^{\prime}\right)$ in $T$.

\section{Sharp Lower Bound on Wiener Polarity Index for $n$-Vertex Trees with a Fixed Number of Segments}

Note that $\mathbb{S} \mathbb{T}_{n, 1}$ consists of only the path graph $P_{n}$, and $\mathbb{S} \mathbb{T}_{n, 2}$ is empty. Thus, we proceed in this note with the assumption $3 \leq s \leq n-1$. Denote by $S_{s}^{n} \in \mathbb{S T}_{n, s}$ the starlike tree with $s-1$ pendent paths of length 1 (see Figure 1). Let $S T_{n, s}^{*} \subset \mathbb{S} \mathbb{T}_{n, s}$ be the class of all $n$-vertex trees with exactly one internal path and $s-1$ pendent paths of length 1 . For the tree(s) having the minimum Wiener Polarity index among all the members of the class $\mathbb{S} \mathbb{T}_{n, s}$, we firstly prove some lemmas.

Lemma 1. Let $n$ and $s$ be positive integers such that $3 \leq s \leq n-1$. If $T \in \mathbb{S T}_{n, s}$ is a tree such that $W_{p}(T)$ is minimum among all the trees of $\mathbb{S} \mathbb{T}_{n, s}$, then $T$ contains at most one pendent path of length greater than 1 .

Proof. Suppose, contrarily, that $P=v_{0}, v_{1}, \ldots, v_{s}$ and $P^{\prime}=$ $v_{0}^{\prime}, v_{1}^{\prime}, \ldots, v_{l}^{\prime}(l, s \geq 2)$ are two pendent paths in $T$, where $d_{v_{0}}=$ $d_{v_{0}^{\prime}}=1$ and $d_{v_{s}}, d_{v_{l}^{\prime}} \geq 3$ (note that the vertices $v_{l}^{\prime}$ and $v_{s}$ may coincide). If $T^{s^{\prime}}=T-\left\{v_{s-1} v_{s-2}\right\}+\left\{v_{s-2} v_{0}^{\prime}\right\}$, then $T^{\prime} \in \mathbb{S} \mathbb{T}_{n, s}$, and we have

$$
W_{p}(T)-W_{p}\left(T^{\prime}\right)=d_{v_{s}}-2>0,
$$

a contradiction to the choice of $T$.

Lemma 1 ensures that the trees $S_{3}^{n}$ and $S_{4}^{n}$ have the minimum $W_{p}$ value in the classes $\mathbb{S T}_{n, 3}$ and $\mathbb{S} \mathbb{T}_{n, 4}$,

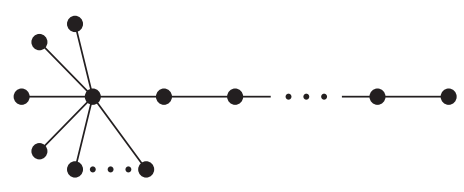

Figure 1: The graph $S_{s}^{n}$.

respectively. Also, it is obvious that the star graph $S_{n}$ gives the minimum $W_{p}$ value (that is, 0) in the class $\mathbb{S T}_{n, n-1}$. Therefore, we proceed with the assumption $5 \leq s \leq n-2$. Denote by $\mathbb{S} \mathbb{T}_{s} \subset \mathbb{S} \mathbb{T}_{n, s}$ the subclass consisting of all starlike trees. Moreover, by Lemma $1, S_{s}^{n}$ attains the minimum $W_{p}$ value in the class $\mathbb{S T}_{s}$. Now, we consider the class $\mathbb{S T}_{n, s} \backslash \mathbb{S} \mathbb{T}_{s}$ where $5 \leq s \leq n-2$.

Lemma 2. Let $n$ and $s$ be positive integers such that $5 \leq s \leq n-2$. If $T \in \mathbb{S T}_{n, s} \backslash \mathbb{S} \mathbb{T}_{s}$ is a tree having minimum $W_{p}$ value among all the members of $\mathbb{S} \mathbb{T}_{n, s} \backslash \mathbb{S} \mathbb{T}_{s}$, then each pendent path of $T$ is of length 1 .

Proof. We contrarily assume that there is a pendent path $P:=v_{0}, v_{1}, \ldots, v_{s}$ of length $s \geq 2$ in $T$, where $d_{v_{0}}=1$ and $d_{v_{s}} \geq 3$. Let $v \in V(T)$ be a branching vertex different from $v_{s}$ and let $u$ be the neighbor of $v_{s}$ lying on the $v_{s}-v$ path. Note that $d_{u} \geq 2$ and that $u$ may coincide with $v$. Let $T^{\prime}=T-\left\{v_{s} u, v_{0} v_{1}\right\}+\left\{v_{0} v_{s}, v_{1} u\right\}$, it can be observed that $T^{\prime} \in \mathbb{S T}_{n, s} \backslash \mathbb{S} \mathbb{T}_{s}$, and we have $W_{p}(T)-W_{p}\left(T^{\prime}\right)=$ $\left(d_{u}-1\right)\left(d_{v_{s}}-2\right)>0$, a contradiction to the choice of $T$.

Theorem 1. Let $n$ and $s$ be positive integers such that $3 \leq s \leq n-2$. If $T \in \mathbb{S} \mathbb{T}_{n, s}$, then

$$
W_{p}(T) \geq n-3,
$$

and the equality sign in (3) holds if and only if either $T \cong S_{s}^{n}$ (see Figure 1) or $T \in S T_{n, s}^{*}$.

Proof. If $T \in \mathbb{S T}_{n, s}$ contains more than one pendent path of length at least 2 , then by the proof of Lemma 1, there exists a tree $T^{*}$ having at most one pendent path of length at least 2 such that $W_{p}(T)>W_{p}\left(T^{*}\right)$. Thus, it is enough to prove the result when $T \in \mathbb{S} \mathbb{T}_{n, s}$ contains at most one pendent path of length at least 2 . In the remaining proof, we assume that $T \in \mathbb{S T}_{n, s}$ has at most one pendent path of length at least 2 .

If either $T \cong S_{s}^{n}$ or $T \in S T_{n, s}^{*}$, then by elementary calculations, one has $W_{p}(T)=n-3$. We apply induction on $s$ to prove the desired result. Note that if $s=3$ or 4 , then by Lemma 1, it holds that $W_{p}(T) \geq n-3$ with equality if and only if $T \cong S_{s}^{n}$. Also, if $s=5$, then by using Lemmas 1 and 2 , we have $W_{p}(T) \geq n-3$ with equality if and only if either $T \cong S_{5}^{n}$ or $T \in S T_{n, 5}^{*}$. Next, suppose that $6 \leq s \leq n-2$ and that the result holds for every $s^{\prime}$ satisfying $3 \leq s^{\prime} \leq s-1$.

Let $P: w_{1}, w_{2}, \ldots, w_{r}$ be a longest path in $T$, where $r \geq 4$. Note that each of the two vertices $w_{2}$ and $w_{r-1}$ has exactly one nonpendent neighbor in $T$. Since $T$ contains at most one pendent path of length at least 2 , at least one of the two vertices $w_{2}$ and $w_{r-1}$ is branching. Without loss of generality, we assume that $w_{2}$ is branching. Let $N\left(w_{2}\right)=\left\{w_{1}, w_{3}, u_{1}, u_{2}\right.$, $\left.\ldots, u_{t}\right\}$ where $t \geq 1$ and $d_{u_{i}}=1$ for every $i \in\{1,2, \ldots, t\}$. Let 
$T^{\prime}=T-\left\{u_{1}\right\}$. Note that $T^{\prime} \in \mathbb{S T}_{n-1, s-1}$ when $t \geq 2$, and $T^{\prime} \in \mathbb{S T}_{n-1, s-2}$ when $t=1$. Hence, by using the inductive hypothesis, we have

$$
\begin{aligned}
W_{p}(T) & =W_{p}\left(T^{\prime}\right)+\left(d_{w_{3}}-1\right) \\
& \geq n-4+\left(d_{w_{3}}-1\right) \\
& \geq n-3 .
\end{aligned}
$$

If $t \geq 2$, then the equality $W_{p}(T)=n-3$ holds if and only if $d_{w_{3}}=2$ and either $T^{\prime} \in S T_{n-1, s-1}^{*}$ or $T^{\prime} \cong S_{s-1}^{n-1}$. If $t=1$, then the equality $W_{p}(T)=n-3$ holds if and only if $d_{w_{3}}=2$ and $T^{\prime} \cong S_{s-2}^{n-1}$ (because in this case, the tree $T^{\prime}$ contains a pendent path of length at least 2). Thus, we conclude that $W_{p}(T) \geq n-3$ with equality if and only if $T \cong S_{s}^{n}$ or $T \in S T_{n, s}^{*}$. This completes the induction and hence the proof.

\section{Sharp Lower Bound on Wiener Polarity Index for $n$-Vertex Trees with a Given Number of Branching Vertices}

Recall that $\mathbb{T}_{n, b}$ is the class of all $n$-vertex trees with $b$ branching vertices, where $1 \leq b \leq(n / 2)-1$. For $b=1$, the star graph $S_{n}$ attains the minimum $W_{p}$ value (see [36]). Thus, throughout this section, we assume $2 \leq b \leq(n / 2)-1$. Note that Lemma 3 may be proved in a fully analogous way to that of Lemma 2.

Lemma 3 (see [35]). Let $b$ and $n$ be positive integers such that $2 \leq b \leq(n / 2)-1$. If $T \in \mathbb{T}_{n, b}$ is a tree having minimum $W_{p}$ value among all the members of $\mathbb{T}_{n, b}$, then every pendent path of $T$ is of length 1.

Let $x_{i, j}$ be the number of edges in a tree $T$ connecting the vertices of degrees $i$ and $j$.

Lemma 4. Let $b$ and $n$ be positive integers such that $2 \leq b \leq(n / 2)-1$. If $T \in \mathbb{T}_{n, b}$ is a tree having minimum $W_{p}$ value among all the members of $\mathbb{T}_{n, b}$ and $x_{i, 1} \neq 0$ for some $i \geq 4$, then $T$ does not contain any pair of adjacent branching vertices.

Proof. Contrarily, suppose that $w, z \in V(T)$ is a pair of adjacent branching vertices and let $v \in V(T)$ be a pendent vertex adjacent to a vertex $u \in V(T)$ of degree at least 4 . Note that $u$ may coincide with either of the vertices $w$ and $z$. If $T^{\prime}=T-\{v u, w z\}+\{w v, v z\}$, then it can be observed that $T^{\prime} \in \mathbb{T}_{n, b}$, and we have

$$
W_{p}(T)-W_{p}\left(T^{\prime}\right)=\sum_{x \in N(u), x \neq v}\left(d_{x}-1\right)+d_{w} d_{z}-2 d_{w}-2 d_{z}+3,
$$

which is positive because of the fact that the function $f(a, b)=a b-2 a-2 b+3$ is strictly increasing in both $a$ and $b$ where $a, b \in(3, \infty]$. Thus, we arrived at a contradiction to the choice of $T$.

Lemma 5. Let $b$ and $n$ be positive integers such that $2 \leq b \leq(n / 2)-1$. If $T \in \mathbb{T}_{n, b}$ is a tree with minimum $W_{p}$ among the trees from $\mathbb{T}_{n, b}$, such that $u v \in E(T)$ with $d_{u}=1$ and $d_{v} \geq 4$, then a tree $T^{\prime} \in \mathbb{T}_{n, b}$ can be obtained from $T$ as $T^{\prime}=T-\{v w\}+\{u w\}$, where $w$ is a nonpendent neighbor of $v$, such that $W_{p}(T) \geq W_{p}\left(T^{\prime}\right)$.

Proof. It holds, as it is easy to see that $T^{\prime} \in \mathbb{T}_{n, b}$. Also, using the facts $d_{w} \geq 2$ and $d_{v} \geq 4$, we have

$$
\begin{aligned}
& W_{p}(T)-W_{p}\left(T^{\prime}\right)=\left(d_{v}-1\right)\left(d_{w}-1\right)+\left(d_{v}-1\right) \\
& \sum_{z \in N(v), z \neq u, z \neq w}\left(d_{z}-1\right)-\left(d_{w}-1\right)-\left(d_{v}-2\right)-\left(d_{v}-2\right) \\
& \quad \sum_{z \in N(v), z \neq u, z \neq w}\left(d_{z}-1\right) \\
& =d_{v} d_{w}-2 d_{v}-2 d_{w}+4+\sum_{z \in N(v), z \neq u, z \neq w}\left(d_{z}-1\right) \geq 0,
\end{aligned}
$$

which implies $W_{p}(T) \geq W_{p}\left(T^{\prime}\right)$.

Lemma 6. Let $b$ and $n$ be positive integers such that $2 \leq b \leq(n / 2)-1$. If $T \in \mathbb{T}_{n, b}$ is a tree having minimum $W_{p}$ value among all the members of $\mathbb{T}_{n, b}$, then every vertex of degree greater than 3 in $T$ has exactly one nonpendent neighbor.

Proof. We contrarily assume that the vertex $u \in V(T)$, with $N(u)=\left\{u_{1}, u_{2}, \ldots, u_{q}, u_{q+1}, \ldots, u_{t}\right\}$, has at least two nonpendent neighbors where $t \geq 4$. We consider the following cases:

Case 1. The vertex $u$ has at least one pendent neighbor.

Without loss of generality, we assume that $d_{u_{i}}=1$ for $1 \leq i \leq q$ and $d_{u_{j}}=d_{j} \geq 2$ for $q+1 \leq j \leq t$. Then, $t-q \geq 2$ because $u$ has at least two nonpendent neighbors. Lemma 4 ensures that $d_{j}=2$ for every $j$ satisfying $q+1 \leq j \leq t$. If $T^{\prime}=T-\left\{u u_{t}\right\}+\left\{u_{t} u_{1}\right\}$, then $T^{\prime} \in \mathbb{T}_{n, b}$ and hence, because of the fact $t-q \geq 2$, we have

$$
W_{p}(T)-W_{p}\left(T^{\prime}\right)=t-q-1>0,
$$

which is a contradiction.

Case 2. The vertex $u$ has nonpendent neighbor.

In this case, we have $d_{u_{i}} \geq 2$ for every $i$ satisfying $1 \leq i \leq t$. Here, Lemmas 3-5 ensure that there is a pendent vertex $v \in V(T)$ having the neighbor $w$ such that $d_{w}=3$ for $d_{u_{i}} \geq 2$, where $1 \leq i \leq t$. Let $u_{1}$ be the neighbor of $u$ that lies on the unique $v-u$ path. If $T^{\prime}=T-\left\{u u_{t}\right\}+\left\{v u_{t}\right\}$, then $T^{\prime} \in \mathbb{T}_{n, b}$, and we have

$$
W_{p}(T)-W_{p}\left(T^{\prime}\right)=\sum_{x \in N(u), x \neq u_{t}}\left(d_{x}-1\right)+\left(d_{u}-2\right)\left(d_{u_{t}}-1\right)-2>0,
$$

which is again a contradiction to the choice of $T$.

Theorem 2. Let $b$ and $n$ be positive integers such that $2 \leq b \leq(n / 2)-1$. If $T \in \mathbb{T}_{n, b}$, then 


$$
W_{P}(T) \geq \begin{cases}n+b-5, & 2 \leq b<\frac{n-1}{3}, \\ 4 b-4, & \frac{n-1}{3} \leq b \leq \frac{n}{2}-1,\end{cases}
$$

and the equality holds if and only if $T \in \mathbb{T}_{1}^{*}$, for $2 \leq b<(n-1 / 3)$, where $\mathbb{T}_{1}^{*}=\{T: T$ is a tree whose every vertex with degree $\geq 4$ has exactly one nonpendent neighbor and each internal path is of length at least 2$\}$, and $T \in \mathbb{T}_{2}^{*}$, for $(n-1 / 3) \leq b \leq(n / 2)-1$, where $\mathbb{T}_{2}^{*}$ is a class of trees with degree sequence $(\underbrace{3,3, \ldots, 3}_{b}, \underbrace{2,2, \ldots, 2}_{n-2 b-2}, \underbrace{1,1, \ldots, 1}_{b+2})$ such that each pendent vertex of $T \in \mathbb{T}_{2}^{*}$ is adjacent to some branching vertex only.

Proof. Denote by $N_{i}$ the number of vertices of degree $i$ in a graph $G$. Let $T$ be a tree that minimizes $W_{p}$ among the class $\mathbb{T}_{n, b}$. Lemma 3 and Lemma 4 conclude that whenever $(n-1 / 3) \leq b \leq(n / 2)-1$, every branching vertex in $T$ has degree 3 such that the vertices of degree 2 are placed between the adjacent vertices of degree 3 in such a way that no two vertices of degree 2 are adjacent if there are adjacent vertices of degree 3. Note that, for $(n-1 / 3) \leq b \leq(n / 2)-1$, we have $N_{3}=b, \quad N_{1}=b+2 \quad$ and $\quad N_{2}=n-2 b-2$. Hence, $W_{p}(T)=4 b-4$.

Now, Lemmas 3-6 conclude that every internal path has a length of at least 2. Also, Lemma 5 ensures that, to obtain minimal graph $T$, either we have to insert the vertices of degree 2 between any vertex of degree 2 and vertex of degree 3 , or we have to add a starlike pendent vertex in such a way that every vertex with degree $\geq 4$ has exactly one nonpendent neighbor that is $T \cong \mathbb{T}_{1}^{*}$. Hence, $W_{p}(T)=n+b-5$, for $2 \leq b<(n-1 / 3)$, which completes the proof.

\section{Data Availability}

The data used to support the findings of the study are available from the corresponding author upon request.

\section{Conflicts of Interest}

The authors declare that they have no conflicts of interest.

\section{References}

[1] N. Trinajstić, Chemical Graph Theory, CRC Press, Boca Raton, FL, USA, 2nd edition, 1992.

[2] B. Basavanagoud, V. R. Desai, and S. Patil, " $(\beta, \alpha)$-connectivity index of graphs," Applied Mathematics and Nonlinear Science, vol. 2, no. 1, pp. 21-30, 2017.

[3] A. Q. Baig, M. Naeem, and W. Gao, "Revan and hyper-Revan indices of octahedral and icosahedral networks," Applied Mathematics and Nonlinear Sciences, vol. 3, no. 1, pp. 33-40, 2018.

[4] M. S. Sardar, S. Zafar, and Z. Zahid, "Computing topological indices of the line graphs of Banana tree graph and Firecracker graph," Applied Mathematics and Nonlinear Sciences, vol. 2, no. 1, pp. 83-92, 2017.
[5] H. Wiener, "Structural determination of paraffin boiling points," Journal of the American Chemical Society, vol. 69, no. 1, pp. 17-20, 1947.

[6] H. Lei, Y. Shi, and J. Yue, "A survey on the Wiener polarity index," MATCH Communications in Mathematical and in Computer Chemistry, vol. 86, no. 2, 2021, in press.

[7] J. Yue, Y. Shi, and H. Wang, "Bounds of Wiener polarity index," in Bounds in Chemical Graph Theory-Basics(MCM NO.19), K. C. Das, B. Furtula, I. Gutman, E. Milovanović, and I. Milovanovi, Eds., pp. 283-302, University of Kragujevac and Faculity of Kragujevac, Kragujevac, Serbia, 2017.

[8] A. Ali, Z. Du, and M. Ali, "A note on chemical trees with minimum Wiener polarity index," Applied Mathematics and Computation, vol. 335, pp. 231-236, 2018.

[9] A. Ali, Z. Du, S. S. Zaineb, and T. Alraqad, "On the second maximum Wiener polarity index of chemical trees of a fixed order," International Journal of Quantum Chemistry, accepted for publication.

[10] A. R. Ashrafi and A. Ghalavand, "Ordering chemical trees by Wiener polarity index," Applied Mathematics and Computation, vol. 313, pp. 301-312, 2017.

[11] L. Chen, T. Li, J. Liu, Y. Shi, and H. Wang, "On the Wiener polarity index of lattice networks," PLoS One, vol. 11, no. 12, Article ID e0167075, 2016.

[12] H. Deng, "On the extremal Wiener polarity index of chemical trees," MATCH Communications in Mathematical and in Computer Chemistry, vol. 66, pp. 305-314, 2011.

[13] Z. Du and A. Ali, "The Alkanes with maximum Wiener polarity index," Molecular Informatics, vol. 37, Article ID 1800076, 2018.

[14] Z. Du and A. Ali, "The inverse Wiener polarity index problem for chemical trees," PLoS One, vol. 13, Article ID e0197142, 2018.

[15] W. Du, X. Li, and Y. Shi, "Algorithms and extremal problem on Wiener polarity index," MATCH Communications in Mathematical and in Computer Chemistry, vol. 62, pp. 235244, 2009.

[16] A. Ghalavand and A. R. Ashrafi, "Ordering chemical unicyclic graphs by Wiener polarity index," International Journal of Quantum Chemistry, vol. 17, no. 119, 2019.

[17] H. Hua and K. C. Das, "On the Wiener polarity index of graphs," Applied Mathematics and Computation, vol. 280, pp. 162-167, 2016.

[18] H. Hosoya, "Mathematical and chemical analysis of Wieners polarity number," in Topology in Chemistry Discrete Mathematics of Molecules, D. H. Rouvray and R. B. King, Eds., p. 57, Horwood, Chichester, UK, 2002.

[19] J. Ma, Y. Shi, Z. Wang, and J. Yue, "On Wiener polarity index of bicyclic networks,” Scientific Reports, vol. 6, 2016.

[20] S. Noureen, A. Ali, and A. A. Bhatti, Towards the Solution of an Extremal Problem Concerning the Wiener Polarity Index of Alkanes, Chaos Solitons Fractals, , in press.

[21] F. Shafiei and A. Saeidifar, "QSPR study of some physicochemical properties of sulfonamides using topological and quantum chemical indices," Journal of the Chemical Society of Pakistan, vol. 39, no. 3, pp. 366-373, 2017.

[22] A. Safari and F. Shafiei, "QSPR models of physicochemical properties of natural amino acids by using topological indices and MLR method," Journal of the Chemical Society of Pakistan, vol. 39, no. 5, pp. 752-757, 2017.

[23] N. Tratnik, "Formula for calculating the Wiener polarity index with applications to benzenoid graphs and phenylenes," Journal of Mathematical Chemistry, vol. 57, no. 1, pp. 370383, 2019. 
[24] Y. Zhang and Y. Hu, "The Nordhaus-Gaddum-type inequality for the Wiener polarity index," Applied Mathematics and Computation, vol. 273, pp. 880-884, 2016.

[25] S. Noureen, A. Ali, and A. A. Bhatti, "On the extremal Zagreb indices of $v$-vertex chemical trees with fixed number of segments or branching vertices, MATCH Commun," MATCH Communications in Mathematical and in Computer Chemistry, vol. 84, pp. 513-534, 2020.

[26] J. A. Bondy and U. S. R. Murty, Graph Theory, Springer, Berlin, Germany, 2008.

[27] E. Estrada and D. Bonchev, "Chemical graph theory," in Handbook of Graph Theory, J. L. Gross, J. Yellen, and P. Zhang, Eds., pp. 1538-1558, CRC Press, Boca Raton, FL, USA, 2nd edition, 2013.

[28] F. Harary, Graph Theory, Addison-Wesley, Reading, MA, 1969.

[29] I. Lukovits and W. Linert, "Polarity-numbers of cycle-containing structures," Journal of Chemical Information and Computer Sciences, vol. 38, no. 4, pp. 715-719, 1998.

[30] H. Lei, T. Li, Y. Shi, and H. Wang, "Wiener polarity index and its generalization in trees," MATCH Communications in Mathematical and in Computer Chemistry, vol. 78, pp. 199212, 2017.

[31] M. Liu and B. Liu, "The second Zagreb indices and Wiener polarity indices of trees with given degree sequences, $\mathrm{MATCH}$ Commun," MATCH Communications in Mathematical and in Computer Chemistry, vol. 67, pp. 439-450, 2012.

[32] H. Deng, H. Xiao, and F. Tang, "On the extremal Wiener polarity index of trees with a given diameter," MATCH Communications in Mathematical and in Computer Chemistry, vol. 63, pp. 257-264, 2010.

[33] H. Deng and H. Xiao, "The maximum Wiener polarity index of trees with $k$ pendants," Applied Mathematics Letters, vol. 23, pp. 710-715, 2010.

[34] B. Liu, H. Hou, and Y. Huang, "On the Wiener polarity index of trees with maximum degree or given number of leaves," Computers and Mathematics with Applications, vol. 60, no. 7, pp. 2053-2057, 2010.

[35] S. Shafique and A. Ali, "On the reduced second Zagreb index of trees," Asian-European Journal of Mathematics, vol. 10, no. 4, Article ID 1750084, 2017.

[36] B. Furtula, I. Gutman, and S. Ediz, "On difference of Zagreb indices," Discrete Applied Mathematics, vol. 178, pp. 83-88, 2014.

[37] I. Gutman, B. Furtula, and C. Elphick, "Three new/old vertexdegree-based topological indices," MATCH Communications in Mathematical and in Computer Chemistry, vol. 72, pp. 617-632, 2014. 